\title{
BMJ Open Attitudes and behaviours of adolescents towards antibiotics and self-care for respiratory tract infections: a qualitative study
}

Meredith KD Hawking, ${ }^{1,2}$ Donna M Lecky, ${ }^{1}$ Pia Touboul Lundgren, ${ }^{3,4}$ Eman Aldigs, ${ }^{5}$ Hind Abdulmajed, ${ }^{5}$ Eleni loannidou, ${ }^{6}$ Demetra Paraskeva-Hadjichambi, ${ }^{7}$ Pauline Khouri, ${ }^{3,4}$ Micaela Gal, ${ }^{8}$ Andreas Ch. Hadjichambis, ${ }^{7}$ Demetrios Mappouras, ${ }^{7}$ Cliodna AM McNulty ${ }^{1}$

To cite: Hawking MKD, Lecky DM, Touboul Lundgren $P$, et al. Attitudes and behaviours of adolescents towards antibiotics and self-care for respiratory tract infections: a qualitative study. BMJ Open 2017;0:e015308. doi:10.1136/ bmjopen-2016-015308

- Prepublication history and additional material are available. To view these files please visit the journal online (http://dx.doi.org/ 10.1136/ bmjopen-2016-015308).

Received 24 November 2016 Revised 27 February 2017 Accepted 5 April 2017
CrossMark

For numbered affiliations see end of article.

Correspondence to

Dr. Donna M Lecky;

Donna.lecky@phe.gov.uk

\section{ABSTRACT}

Background To understand attitudes and behaviours of adolescents towards antibiotics, antimicrobial resistance and respiratory tract infections.

Design Qualitative approach informed by the Theory of Planned Behaviour. Semi-structured interviews and focus groups were undertaken. We aimed to inform the development of an intervention in an international setting to improve antibiotic use among adolescents; therefore on completion of thematic analysis, findings were triangulated with qualitative data from similar studies in France, Saudi Arabia and Cyprus to elucidate differences in the behaviour change model and adaptation to diverse contexts.

Setting 7 educational establishments from the south of England.

Participants 53 adolescents (16-18 years) participated in seven focus groups and 21 participated in interviews. Results Most participants had taken antibiotics and likened them to other common medications such as painkillers; they reported that their peers treat antibiotics like a 'cure-all' and that they themselves were not interested in antibiotics as a discussion topic. They demonstrated low knowledge of the difference between viral and bacterial infections.Participants self-cared for colds and flu but believed antibiotics are required to treat other RTIs such as tonsillitis, which they perceived as more 'serious'. Past history of taking antibiotics for RTIs instilled the belief that antibiotics were required for future RTIs. Those who characterised themselves as 'nonscience students' were less informed about antibiotics and AMR. Most participants felt that AMR was irrelevant to them and their peers. Some 'non-science' students thought resistance was a property of the body, rather than bacteria.

Conclusion Addressing adolescents' misperceptions about antibiotics and the treatment of RTIs using a behaviour change intervention should help improve antibiotic awareness and may break the cycle of patient demand for antibiotics to treat RTIs amongst this group. Schools should consider educating all students in further education about antibiotic usage and AMR, not only those taking science.
Strengths and limitations of this study

- The use of focus groups gave researchers insight into peer group interaction and shared viewpoints, whereas interviews allowed participants to share personal views more freely in a private setting.

- Triangulation of the data from this study against similar studies in other countries confirms the transferability of the findings to other settings.

- Recruitment of participants from non-healthcare settings provides insight into views and behaviours among healthy adolescents.

- Although schools were chosen to maximise diversity, researchers could only influence the selection of participants within educational establishments by providing guidelines and a sampling matrix to educators.

- Adolescents not enrolled in education were not included in the study and further research would be required to explore perceptions among this group.

\section{BACKGROUND}

The increase in antimicrobial resistance (AMR) has the potential for devastating impact on public health and the provision of healthcare worldwide. There are an estimated 25000 deaths per year in Europe from antibiotic-resistant bacterial infections. ${ }^{1}$ The majority of antibiotics are prescribed in primary care, and much of this prescribing is for self-limiting upper respiratory tract infections (URTIs) ${ }^{2}$ for which antibiotics are often unnecessary, ${ }^{3}$ with individuals more likely to develop bacterial resistance to that antibiotic after use. ${ }^{4}$ Moreover, patient expectation for antibiotics in primary care is linked to higher prescribing. ${ }^{56}$ Therefore, WHO has stressed the need to raise awareness of the responsible use of antibiotics and the threat of AMR among the general public. ${ }^{7}$ However, in spite of numerous interventions targeting 
the public and healthcare professionals, antibiotic use is increasing worldwide. ${ }^{8}$

A large European survey has shown that those aged 15-24years are the highest users of antibiotics and are more likely than other age groups to take them for URTIs. ${ }^{9}$ In addition, research in the UK found that younger members (15-34 years) of the general public have lower knowledge around antibiotics, AMR and how to care for self-limiting infections than older groups. ${ }^{10}$ Qualitative research undertaken in healthcare settings has investigated patient perceptions of antibiotics and AMR of those $>18$ years of age. ${ }^{11-13}$ They found that adult patients see antibiotic resistance as a societal or hospital problem due to the widespread overuse of antibiotics, but not as something that could affect them personally, and they do not believe that their individual antibiotic use could contribute to or help resolve the issue. ${ }^{11-13}$ Research on parental perceptions of RTIs and antibiotics in regards to their children have also been undertaken; ${ }^{14}{ }^{15}$ however, to date, older adolescents have attracted less attention from qualitative researchers as a unique group of participants. These older adolescents (16-18 years) may be a distinct group of antibiotic users for investigation-unlike younger children, they may be in control of their own medicines ${ }^{16}$ and responsible for their use. Adolescents are keen to distance themselves from their parental influence and create their own self-image, ${ }^{17}$ so parents may play a limited role in the day-to-day management of medications for common infections such as RTIs. However, as adolescents become more independent, their health-related behaviour may be governed by social norms influenced by their peers, parents and educators. We decided to investigate this complex behavioural picture among those aged 16-18years as they are in education and therefore school-based, targeted interventions might be an effective strategy to increase appropriate antibiotic use.

Behavioural interventions have been shown to be effective at improving antibiotic-related behaviour among parents consulting on behalf of their children for RTIs, ${ }^{18}$ but there is little research on interventions targeting adolescents. In order to be successful, any intervention must be theoretically driven, evidence based and adapted to the local context. Qualitative inquiry provides a robust methodology for exploring the complex views and behaviours of this target group. Furthermore, we aimed to triangulate the findings of this study with country partners of the e-Bug school educational project to inform transferability of the findings to other settings. e-Bug is a publicly funded international collaborative public health project which aims to improve school students' knowledge and behaviours towards antibiotics and vaccinations. ${ }^{19}$

\section{METHODS}

\section{Study design}

We conducted in-depth, semi-structured interviews with older adolescents (16-18 years) in both an individual interview and focus group setting at educational
Invite sent to schools ( $\mathbf{n = 3 7 )}$

First stage schools consent to take part $(\mathbf{n}=\mathbf{3})$

Characteristics of first stage schools assessed

Unrepresented schools approached $(n=6)$

Second stage schools consent to take part $(n=4)$

Adolescents invited to take part by coordinators

Characteristics of adolescents assessed

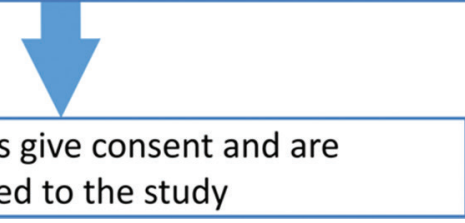

Figure 1 Recruitment flowchart.

establishments in the south of England. The Theory of Planned Behaviour (TPB) ${ }^{20}$ was used as a theoretical basis to inform the development of the topic guide to ensure the topics raised covered multiple factors that influence behaviour. The TPB was used to shape the questions asked in the interview, and therefore influenced the topics that emerged. However, the TPB was not used to directly structure the thematic analysis. This was to allow for an inductive approach, where the themes and thematic framework are shaped by the data, and to allow for any new constructs that had arisen directly from the interviews. The TPB aims to predict and explain human behaviour in specific contexts and identifies intention as the central factor to performance of a specific behaviour. The TPB suggests that individuals are more likely to intend to perform a behaviour (take antibiotics) if they have a positive attitude towards it, perceive social pressure from others to perform the behaviour (subjective norms) and perceive that the performance of the behaviour is within their control (perceived behavioural control). Exploration of these constructs and the data that results provides a practical basis for the development of an intervention to change attitudes, norms and behavioural intention.

\section{Setting and participants}

A two-stage purposive sampling technique was used to recruit schools and colleges (figure 1). All registered educational establishments teaching those aged 16-18years in 


\begin{tabular}{|c|c|}
\hline Topic & Probes \\
\hline History of taking antibiotics & General experience \\
\hline Perceptions of antibiotics & Usage, positives, negatives \\
\hline $\begin{array}{l}\text { Management of colds/ } \\
\text { influenza }\end{array}$ & Self-care, use of medications \\
\hline Consulting for RTIs & $\begin{array}{l}\text { Decision to consult, advice, } \\
\text { expectations }\end{array}$ \\
\hline Others' views on antibiotics & $\begin{array}{l}\text { Friends, parents, teachers, } \\
\text { media }\end{array}$ \\
\hline $\begin{array}{l}\text { Antimicrobial resistance } \\
\text { (AMR) }\end{array}$ & $\begin{array}{l}\text { Knowledge, importance, } \\
\text { responsibility }\end{array}$ \\
\hline Others' views on AMR & $\begin{array}{l}\text { Friends, parents, teachers, } \\
\text { media }\end{array}$ \\
\hline Sources of information & $\begin{array}{l}\text { Friends, family, media, } \\
\text { school, others }\end{array}$ \\
\hline
\end{tabular}

Gloucestershire were invited to participate by letter and telephone. Additionally, all educational establishments serving those aged 16-18years on the e-Bug mailing list were invited in order to geographically widen the sampling area and include establishments based in areas with differing levels of deprivation. Participating establishments were also selected to represent different school and college types, selection policies and governance structures. An educator was identified as the 'study coordinator' in each school and was given written guidelines to inform their selection of participants: 16-18-year-old adolescents were purposively sampled according to gender and area with the aid of a sampling matrix, aiming to achieve a diverse population and range of views. Informed written consent was obtained from all participating schools and adolescents, and parents could refuse child inclusion via 'opt-out' forms. Participation was voluntary, therefore we do not have information on those students who did not wish to take part.

\section{Data collection}

The semi-structured topic guide was piloted with five participants and reviewed by the research team prior to data collection commencement. It included open questions and follow-up probes to elucidate views on antibiotics, AMR and RTIs (table 1). Care was taken to ensure that it did not constrain discussions and allowed new topics to emerge.

Interviews and focus groups were held between March and July 2013 in a private classroom or school office. Data collection was undertaken by trained, experienced female qualitative researchers (MKDH and DML) who were currently employed by the e-Bug project. Interviewers introduced themselves as researchers working for Public Health England and e-Bug, with an interest in participants' views on antibiotics. Interviews and focus groups began with confirmation that it was not a test of knowledge and participants were assured anonymity and confidentiality. Educators oversaw organisation and introductions, but were not present during data collection sessions. Researchers had no previous contact with the participants prior to the interview or focus group.

Individual interviews lasted $20-40 \mathrm{~min}$. Focus groups ran for 1 hour and usually consisted of between of 5 and 10 participants. Care was taken to allow and encourage all participants to express their views. Field notes made after each session included impressions and additional topics that had emerged during data collection. Sessions were audio recorded, pseudo-anonymised and transcribed verbatim. Interviews progressed until theoretical data saturation had occurred and no new themes were arising, after discussion and agreement within the research team. All data were encrypted, stored and handled according to data protection regulations. There was no financial reward for establishments or participants; students who took part received a certificate. Repeat interviews were not undertaken and transcripts were not returned to participants for comment.

\section{Data analysis and interpretation}

Inductive thematic analysis of the data was undertaken in six stages, as outlined below. ${ }^{21}$ Due to the unbalance of gender in our sample, data were analysed according to gender and then combined when the themes were found to be common to both female and male participants. First, the transcripts were read by researchers for accuracy, familiarisation and immersion, then a subset (10\%) was annotated with codes by MKDH and DML. Initial codes were then discussed with all researchers to resolve any discrepancies. All codes were derived from the data and a coding schedule was developed for common coding between researchers. Care was taken not to lose the context of a code by coding too narrowly. Codes were then grouped into related categories and sorted into a draft framework of six main themes, which was discussed and agreed between all researchers. Themes were revised iteratively as the fieldwork and analysis progressed. ${ }^{21}$ Use of NVivo software (V.10) facilitated data organisation. Once coding was complete, the one sheet of paper method was used to clarify findings within, and between, themes. ${ }^{22}$ Finally, the results were collated and summarised, where links between the themes were reviewed and finalised, and characteristic quotes identified to illuminate the data. Codes provided with each quote highlight participant characteristics (IS=individual student interview, $F G=$ focus group participant, $F=f e-$ male, $\mathrm{M}=$ male, $\mathrm{Ur}=$ urban, $\mathrm{Ru}=\mathrm{rural}, \mathrm{Sc}=$ science student, $\mathrm{NSc}=$ non-science student $)$.

\section{Triangulation}

On analysis completion, we triangulated the study findings with qualitative data derived using the same topic guide, translated and adapted for local use, from similar samples of those aged 15-18years from schools and an adolescent healthcare centre, in e-Bug partner countries (France $n=21$, Saudi Arabia $n=48$ and Cyprus n=9). The analysis methodology was the same in all four countries, following the structure for thematic analysis as outlined 
above, using a shared coding manual and thematic framework. Findings were translated to English by researchers in each country alongside discussions to ensure culturally specific meanings were retained. Once country -specific analyses were complete, the summary reports were circulated and discussed in detail by all researchers, highlighting comparisons between the findings. Finally, the results for all countries were collated and summarised at a face-to-face meeting, where links between the themes were reviewed and finalised. The aim was to shed light on our findings and to assess their transferability across differing contexts. These countries represent the most diverse range of healthcare systems, antibiotic provision and usage across the e-Bug partner countries, and we used this approach to inform and assess the need for country-specific adaptations to any potential behavioural intervention. For more detailed information about the samples and methods these countries used, please see online supplementary file 1 .

\section{RESULTS}

\section{Final sample}

In total, 74 adolescents took part in the study at seven educational establishments in three counties (Berkshire, Gloucestershire and Essex) in the south of England; 53 participated in seven focus groups and 21 were interviewed (table 2). $24 \%$ of participants were male and $76 \%$ were female. Three of the educational establishments were in rural locales and four in urban settings, with average district deprivation index levels ranging from 10.9 to $22.9 .^{23}$ Participants were recruited from three further education colleges and four sixth form schools, including one Local Education Authority-controlled

\begin{tabular}{|c|c|c|c|c|c|c|}
\hline & \multicolumn{2}{|c|}{ Interviews } & \multicolumn{2}{|c|}{$\begin{array}{l}\text { Focus } \\
\text { groups }\end{array}$} & \multicolumn{2}{|c|}{ Total } \\
\hline & $\mathbf{n}$ & $\%$ & $\mathrm{n}$ & $\%$ & $\mathrm{n}$ & $\%$ \\
\hline \multicolumn{7}{|l|}{ Gender } \\
\hline Female & 14 & 66 & 42 & 79 & 56 & 76 \\
\hline Male & 7 & 33 & 11 & 21 & 18 & 24 \\
\hline \multicolumn{7}{|l|}{ Subject under study } \\
\hline Biology/science & 7 & 33 & 12 & 22 & 19 & 26 \\
\hline Health and social care & 8 & 38 & 21 & 40 & 29 & 39 \\
\hline All other subjects & 6 & 29 & 20 & 38 & 26 & 35 \\
\hline \multicolumn{7}{|l|}{ School type } \\
\hline $\begin{array}{l}\text { Further education } \\
\text { college }\end{array}$ & 8 & 38 & 21 & 40 & 29 & 39 \\
\hline Sixth form & 13 & 62 & 32 & 60 & 45 & 61 \\
\hline \multicolumn{7}{|l|}{ Location } \\
\hline Rural & 6 & 29 & 25 & 47 & 31 & 41 \\
\hline Urban & 15 & 71 & 28 & 53 & 43 & 58 \\
\hline
\end{tabular}

comprehensive, two comprehensive academies and one selective academy. After giving consent, three students dropped out due to illness absence on the day of the interviews, meaning that one focus group only had three members.

\section{Main findings}

Understanding and perceptions of antibiotics

The majority of adolescents had previously taken antibiotics and believed them to be effective for treating infections. Some expressed positive views on antibiotics.

It just feels like you're doing something to make yourself better. So if you're put on antibiotics it's like you're near the end of whatever's wrong with you. (C1FG1,F1,Ru,NSc)

Most participants did not perceive antibiotics to be specifically for bacterial infections and some felt they were no different from other medications such as painkillers, and talked in general terms about medications when they had been asked about antibiotics. Some participants described avoiding antibiotics and painkillers because they were concerned that they would become reliant on them or felt that they were harmful.

It's like a subconscious thing that you always think it's going to be slightly harmful if you take it [antibiotic]. (C7IS3,M,Ur,Sc)

I try not to rely on painkillers and tablets because I feel like my body is going to get used them and then it just won't be able to get better on its own. (C5IS2,F,Ur,NSc)

A small minority of participants directly confused painkillers and antibiotics, or weren't quite sure of the difference between these two groups of medicines.

Is that when you go to the doctors they give you antibiotics? It's paracetamol. Is paracetamol an antibiotic? (C3IS1,F,Ru,NSc)

Like what are antibiotics? [laugh] 'cause I know I've taken like paracetamol and things, I know that's not antibiotics is it? (C3FG3,F2,Ru,NSc)

There was some misunderstanding about the difference between viral and bacterial infections, and those who were aware of the distinction were mostly science students.

Because you go to doctors and you feel like, oh you have got a sore throat, earache, whatever and they're like oh it's a virus, so you get nothing and you go out and it's just like 'oh it's a virus again' but I didn't know the difference between a virus and an infection. (C5IS3,F,Ur,NSc)

I recently spent my work experience, a week at a GPs, and the amount of people who came in asking for antibiotics, it's remarkable! And a lot of the time, it was a viral infection, so they didn't need them. (C7IS$2, \mathrm{M}, \mathrm{Ur}, \mathrm{Sc})$ 
Perceptions and treatment of RTIs

Adolescents grouped different types of uncomplicated RTIs according to their perceived severity, and this categorisation affected their intentions to take antibiotics. Colds and influenza were seen as simple infections that could be treated at home with 'natural' remedies such as honey, taking comfort measures such as drinking hot drinks and resting, or over-the-counter medications such as painkillers. General practitioner (GP) consultations would only be considered if symptoms persisted or were more serious than their normal cold symptoms. A few adolescents indicated that they would expect antibiotics in this case, especially if they had taken them for the same kind of illness before.

I know I had the illness, I know what it feels like and I know taking antibiotics is important because I won't get it again, well hopefully. (C2IS3,F,Ru,Sc)

Other expectations were to receive a clear diagnosis, reassurance and advice from their doctor. RTIs such as tonsillitis and sinusitis were seen as more serious infections that required clinical intervention and would require antibiotics. A few adolescents attributed feeling better during past RTI illnesses to the antibiotic treatment they took, and therefore believed that antibiotics are an effective treatment for RTIs, as exemplified by the following participant:

This was a sinus infection. The doctor didn't, he said that it might help, because it was like viral or bacterial and stuff like that, which I do not really know about. I think if it is viral you are not supposed to have antibiotics and he wasn't sure whether it was viral or bacterial so he gave me antibiotics. After the first week I was still not better, but then after the second week I was better, so taking them must work. (C5IS1,F,Ur,NSc)

\section{Perceptions towards parents and peers}

Most participants thought that their peers take a lot of antibiotics and treat them as a quick fix, a 'cure-all', like painkillers.

They just think it's just like any other medicine that they are taking. (C2IS1,M,Ru,NSc)

Most adolescents felt that their peers do not think about, discuss or worry about AMR, or feel it is relevant to their age group. Antibiotics were not a popular or priority topic of discussion in their peer group.

It's [antibiotics] not something that you talk about, if that makes sense, [laughter] don't just strike up a conversation about it, we just don't. (C4IS4,F,Ur,NSc)

Parents were described as having an advisory role whereby they could influence intentions to take antibiotics, either by ensuring they were used 'only when necessary' or were occasionally reported as encouraging use.
My parents just think they're a means to an end really I suppose, they're to be used when necessary. (C3IS2,F,Ru,Sc)

Some participants reported feeling that their parents were less knowledgeable about antibiotics than themselves.

They're not the smartest people [slight laugh] so they just think 'if you just take them [antibiotics] you'll feel fine', I mean, 'I've taken them I feel fine'. So it's just trying to explain that to them. (C7IS1,M,Ur,Sc)

\section{Antimicrobial resistance}

In general, the majority of adolescents had poor understanding of AMR. A small minority of students in the individual interviews did not know what the term 'antibiotic resistance' meant-one interviewee thought that antibiotic resistance referred to 'people against antibiotics'. With the exception of those who characterised themselves as 'science students', many thought that the 'body or person becomes resistant' to antibiotics, as exemplified by the following excerpts.

I suppose if you really had, a really serious illness and then you couldn't treat it, because you're immune to one of the tablets, then it could have an effect. (C1IS3,F,Ru,NSc)

Isn't that where your body gets, what's it called? Um, too used to antibiotics so that they no longer work. (C2IS2,M,Ru,NSc)

However, despite this lack of knowledge, after prompting, some of those who had incorrect understanding or had not heard of AMR went on to display basic knowledge of resistance in non-scientific terms, such as 'antibiotics might not work for you next time you take them if you haven't taken them properly' or 'overuse of antibiotics means that they may not work in the future', which could act to make them less likely to want to take antibiotics too often. In contrast, students who demonstrated more knowledge of AMR, mostly science students, felt that it was an important issue and awareness should be increased among the general public, as these quotes demonstrate:

I think that's [antibiotic resistance] going to be increasingly important. If we're creating more resistant bacteria we're going to have to try and make new antibiotics, and what do we do when we can't really do anything else? (C3IS2,F,Ru,Sc)

Sooner or later we're going to run out of antibiotics that work, and it's going to take a lot of time and a lot of money to find new ones. And it's probably inevitable, but it could be slowed, that ... if people aren't aware of antibiotic resistance then that situation may come faster. (C7IS2,M,Ur,Sc)

Some participants expressed the view that it is the GP's responsibility to prescribe appropriately and monitor antibiotic usage, and it is not their concern. 
I don't really worry about it, because I think the doctor knows if they're going to give it to you whether it's going to slightly become resistant. [...] I think they'll monitor how much you have. I don't think we have to worry about it ourselves, the patients. (C1FG1,F2,Ru,NSc)

\section{Results: comparison between countries}

Our findings aligned with the main findings from the Cypriot, French and Saudi Arabian samples, with a few exceptions. Interviewed adolescents in the UK, Saudi Arabia and France confused painkillers and antibiotics; however, this was not the case with participants in Cyprus. Adolescents from Saudi Arabia and Cyprus reported obtaining antibiotics over the counter without a prescription, and this was seen as a very common behaviour among peers; however, this is not possible in the UK or France. As with English participants, previous use of antibiotics in Cyprus and Saudi Arabia led to an increased expectation for antibiotics for RTIs. The easy accessibility of antibiotics over the counter in these countries seemed to increase this effect-antibiotics were described as 'candies' and used for a variety of reported symptoms. Finally, like in the UK, parents had an influence over antibiotic-taking behaviour. This was particularly prominent in Saudi Arabia, where parents more commonly encouraged the use of antibiotics in contrast to the UK findings where parents were mainly described as discouraging use, unless they felt that antibiotics were really necessary.

\section{DISCUSSION}

\section{Statement of principal findings}

This study found that, with the exception of some science students, adolescents in educational establishments in England have low knowledge about antibiotics and AMR, and do not feel that these topics are important or relevant to themselves or their peer group. Adolescents are confident to self-care for colds and influenza, but think other types of RTIs such as tonsillitis and sinusitis need antibiotics, a perception that is reinforced by previous experience of receiving antibiotics for infections. Among their peers, they perceive overuse of antibiotics and a lack of concern about AMR. Parents have an advisory role, acting to limit or encourage use. Comparison with data from other countries confirms very similar findings, but greater reported overuse of antibiotics in countries where they are available 'over the counter' without prescription; other countries also had differing levels of parental influence.

\section{Strengths and limitations of this study}

Our final sample was large, with participants from a diverse selection of schools and areas; however, our sampling strategy did result in an unequal gender balance, with more females taking part. It may be that males were less likely to volunteer or the study format was more attractive to females. Selection of participants was governed by the study coordinator (usually an educator) in the school, which we were not able to control; however, establishments were selected carefully to represent different educational types, deprivation levels and geographical areas, and study coordinators were provided with guidelines and a sampling matrix to follow. To assess the impact of gender in the sample, we initially analysed according to gender and found there were no thematic differences between males and females, and therefore combined the findings, so this may not be an important drawback. Triangulation of the data against findings from other countries within the e-Bug project that undertook similar studies allows us to be more confident in the comprehensiveness and transferability of our findings to other 16-18-year-old adolescents in education in the UK and abroad. Further research is required to understand the views of older adolescents who are not enrolled in education.

Adolescents aged 16-18years were recruited from schools regardless of their past exposure to antibiotics or health status, making the results of this study more applicable to healthy adolescents in everyday contexts compared with previous studies that have recruited adult participants from healthcare settings alone. ${ }^{11-13}$ The perceptions and reported behaviours of healthy adolescents may differ, however, from those who are recruited when they are currently experiencing an infection or taking antibiotics. Our use of multiple data collection methods allowed a comprehensive approach: undertaking individual interviews allowed for more candid sharing of information and discussion of more personal beliefs and feelings, and gave quieter students a better chance to voice their views, helping to eliminate difficulties adolescents can have discussing their health, ${ }^{24}$ and concerns about confidentiality. ${ }^{25}{ }^{26}$ On the other hand, the dynamic interaction of focus groups provided insight into shared viewpoints and social norms. ${ }^{27}$ Care was taken to encourage discussion by introducing focus group 'rules', distancing the sessions from examination-type connotations and exclusion of educators from the room.

\section{Comparison with other research}

Misperceptions about antibiotics and AMR have been found in previous qualitative research with adults. ${ }^{11-13} 2829$ The development and the resolution of AMR are seen as the responsibility of other people, hospitals and doctors who overuse antibiotics, which is mirrored in our study. Additionally, lack of understanding around the type of infection that antibiotics should be used for has been shown among this age group and others in other countries. ${ }^{6} 9$ 30-33 In one such study, $38 \%$ believed that antibiotics work against viral infections. ${ }^{6}$ We found that young people believe antibiotics are required to treat what they perceive as 'serious' URTI infections, such as sinus infections and tonsillitis. However, antibiotics have little clinical benefit in these conditions. ${ }^{3}$ Patient expectations for antibiotics within a primary care consultation have been shown to affect whether or not antibiotics are prescribed $^{56}$ and this can have a medicalising effect, 
leading to greater expectation for antibiotics for future episodes of similar infections, ${ }^{5}$ which was expressed by many of our adolescents. Receiving a prescription is a positive and valued action that signals that the consultation has reached a conclusion. ${ }^{34}$ Patient expectations for antibiotic prescriptions and their positive views towards them, as seen in our study, may be linked to the perceived need for action, to ensure 'something is being done' which may be especially important for infections perceived as 'severe'. In connection with a perceived lack of responsibility for the preservation of antibiotics, this desire to 'do something' about the infection could drive antibiotic use among this age group.

Research has shown that painkillers are seen as an essentially harmless 'ordinary, everyday medicine' by young teenage women. ${ }^{35}$ Young adolescents are often responsible for taking their own medication, for example, in a survey of school students, $22 \%$ of students aged 11-13years reported that they were allowed to use pain medications without asking for permission, and this increased significantly with age. ${ }^{16}$ A lack of understanding of the difference between these pain medications and antibiotics, as demonstrated in our study, may have negative impacts on antibiotic use and prescription expectation.

In a recent qualitative study with Swedish teenagers aged 16-19 years, students argued that the effect of paracetamol declined if it was used too often. ${ }^{17}$ This belief is similar to basic understandings of AMR seen in our study. The perception that resistance is a property of the body echoes similar qualitative research undertaken with adults recruited from healthcare settings. ${ }^{11-132829}$

Adolescents in the study said that they would not discuss antibiotics with their friends and did not think the topic of antibiotics or AMR was relevant or interesting to them. Holmström and colleagues ${ }^{17}$ found that Swedish adolescents held similar views about pain medication: it was considered a 'non-issue' and was not discussed. However, in other health-related areas peers have been shown to have an influential role. ${ }^{36}$

Low knowledge of antibiotics has been linked to low educational attainment in studies of adults; ${ }^{30}$ however, the findings in our study suggest that engaging in post-16 science education provides students with a well-rounded knowledge in relation to antibiotics and AMR. This suggests the impact of education may be more nuanced-it would be possible to achieve a high level of educational attainment following a non-scientific pathway in further education and therefore have a poor understanding of antibiotics and AMR. On the other hand, our findings suggest that increasing education adapted to different pathways around antibiotics in further education may help young adults to engage with the topic and act to protect antibiotics for future generations by increasing their intentions to take antibiotics less and only when necessary.

\section{Implications for clinical practice and policymakers}

The perceived need and expectation for antibiotics for the treatment of RTIs by young people should be taken into account by clinicians when adolescents consult, remembering that adolescence is a key time for the development of lifelong health behaviours and decision-making. Provision of reassurance and information about self-care for RTIs ${ }^{37}$ has been shown to be highly satisfactory to patients in this scenario and should therefore be promoted for this age group. It may be useful to address the differences between painkillers and antibiotics with adolescent patients to ensure antibiotics are treated appropriately and to address any concerns about the body becoming reliant on or resistant to antibiotics.

The lack of concern shown by young people for AMR, unless addressed, could contribute to irresponsible use of antibiotics and demand for antibiotics when this age group consult. As the more knowledgeable 'science' students in the study believe it is their personal responsibility to help control AMR, peer-to-peer interventions that promote interaction between science students and those not studying science subjects could raise the profile of antibiotic resistance and situate it as an important and relevant topic for young adults to be concerned with. According to our study, studying science post- 16 years may provide adolescents with more rounded knowledge about antibiotics and AMR that could influence their future health-related behaviour.

\section{Implications for future research}

Our findings demonstrate that there are misperceptions about antibiotics and how to treat RTIs among older adolescents currently enrolled in education in England, which goes some way to explaining the high reported use of antibiotics among this age group seen in previous research. Further research is required to assess perceptions among adolescents who are not in education to assess transferability of the findings to these groups. As our study suggests that young adults in educational environments are a good target group for behavioural interventions, peer-to-peer education and interaction between those studying different subject types could be a key target for intervention development and evaluation. Triangulation with contrasting international contexts demonstrated that a common intervention model would be relevant. While many perceptions are shared among adolescents in different countries, some country-specific adaptation of any intervention would be necessary. The e-Bug team has used the findings to develop a behaviour change intervention for schools and young people. For more information on the development of the intervention, visit the e-Bug website. ${ }^{19}$

\footnotetext{
Author affiliations

${ }^{1}$ Department of Microbiology, Public Health England Primary Care Unit, Gloucestershire Royal Hospital, Gloucester, UK

${ }^{2}$ Centre for Primary Care and Public Health, Barts and The London School of Medicine and Dentistry, Queen Mary University of London, London, UK
} 
${ }^{3}$ Department of Teaching and Research in General Practice, University of Nice Sophia Antipolis, Nice, France

${ }^{4}$ Département de Santé Publique, Hôpital de l'Archet 1, Nice, France

${ }^{5}$ Department of Medical Microbiology and Parasitology, College Of Medicine, King Abdulaziz University, Jeddah, Saudi Arabia

${ }^{6}$ Cyprus Centre for Environmental Research and Education, Limassol, Cyprus ${ }^{7}$ Government of the Republic of Cyprus Ministry of Education and Culture, Nicosia, Cyprus

${ }^{8}$ Department of Population Medicine, Cardiff University School of Medicine, Cardiff, UK

Acknowledgements A warm thanks to all of the participants and to their educators for their help in facilitating the study in each school, and to the youth health centre Carrefour Santé Jeunes and General Council in Nice, France.

Contributors MH was the main researcher, wrote the protocol, recruited schools, interviewed participants in the UK, coded the data from the UK, Cyprus and Saudi Arabia and led analysis of the data and writing the manuscript. DL discussed the protocol, supervised the project, interviewed participants in the UK, double coded the data and edited the manuscript. PTL facilitated the project in France and coded and analysed the French data with PK who also carried out the interviews in France. EA and HA facilitated the project in Saudi Arabia, collecting the data and translating the interviews. $\mathrm{DH}, \mathrm{AChH}$ and DM facilitated the project in Cyprus. El interviewed the participants and organised transcript translation. MG was involved in protocol development and commented on methodology throughout data collection. CMcN led the overall project, advising on protocol, methodology, analysis and interpretation. All authors reviewed and commented on the protocol and the manuscript.

Disclaimer The funding body had no role in the design, execution, analysis or reporting of the research.

Competing interests CM leads the e-Bug project development and writes evidence-based antibiotic guidance for primary care and leads the Royal College of General Practitioners (RCGP) TARGET (Treat Antibiotics Responsibly, Guidance and Education Tools) antibiotic toolkit development including a patient leaflet encouraging delayed/back-up prescribing. She is a past member (2008-2016) of the Advisory Committee on Antimicrobial Resistance and Healthcare Associated Infection (ARHAl). All other authors were involved in the e-Bug project in their respective countries at the time of the research.

Ethics approval Public Health England, Governmental Disclosure and Barring Service (DBS). Internal Public Health England review only was required as participants were not recruited through the National Health Service (NHS) and all UK participants were $>16$ years of age. The Department of Education was consulted and the study was referred to the Governmental Disclosure and Barring Service (DBS). The study was undertaken in full accordance with the DBS regulations.

Provenance and peer review Not commissioned; externally peer reviewed.

Data sharing statement Unpublished data from the study can be availed upon request from DML.

Open Access This is an Open Access article distributed in accordance with the Creative Commons Attribution Non Commercial (CC BY-NC 4.0) license, which permits others to distribute, remix, adapt, build upon this work non-commercially, and license their derivative works on different terms, provided the original work is properly cited and the use is non-commercial. See: http://creativecommons.org/ licenses/by-nc/4.0/

(c) Article author(s) (or their employer(s) unless otherwise stated in the text of the article) 2017. All rights reserved. No commercial use is permitted unless otherwise expressly granted.

\section{REFERENCES}

1. European Centre for Disease Control. The bacterial challenge: time to react. 2009 http://www.ecdc.europa.eu/en/publications/Publications/ 0909 TER The Bacterial Challenge Time to React.pdf

2. Public Health England. The english surveillance programme for antimicrobial utilisation and resistance report 2014. 2014 https:// www.gov.uk/government/uploads/system/uploads/attachment_data/ file/362374/ESPAUR_Report_2014_3_.pdf

3. National Institute for Health and Clinical Excellence. Respiratory tract infections - antibiotic prescribing: Prescribing of antibiotics for selflimiting respiratory tract infections in adults and children in primary care. 2008 http://www.nice.org.uk/nicemedia/live/12015/41323/ 41323.pdf
4. Costelloe C, Metcalfe C, Lovering A, et al. Effect of antibiotic prescribing in primary care on antimicrobial resistance in individual patients: systematic review and meta-analysis. BMJ 2010;340:c2096.

5. Butler CC, Rollnick S, Pill R, et al. Understanding the culture of prescribing: qualitative study of general practitioners' and patients perceptions of antibiotics for sore throats. BMJ 1998;317:637-42.

6. McNulty CA, Nichols T, French DP, et al. Expectations for consultations and antibiotics for respiratory tract infection in primary care: the RTI clinical iceberg. $B r J$ Gen Pract 2013;63:429-36.

7. World Health Organisation. Combating antimicrobial resistance, including antibiotic resistance. 2014 http://apps.who.int/gb/ebwha/ pdf files/EB134/B134_R13-en.pdf

8. Laxminarayan R, Duse A, Wattal C, et al. Antibiotic resistance-the need for global solutions. Lancet Infect Dis 2013;13:1057-98.

9. European Commission. Special eurobarometer 407: antimicrobial resistance. secondary special eurobarometer 407: antimicrobial resistance. 2013 http://ec.europa.eu/public_opinion/archives/ebs/ ebs_407_en.pdf

10. McNulty CA, Lecky DM, Hawking MK, et al. How much information about antibiotics do people recall after consulting in primary care? Fam Pract 2016;33:395-400.

11. Hawkings NJ, Wood F, Butler CC. Public attitudes towards bacterial resistance: a qualitative study. J Antimicrob Chemother 2007;59:1155-60.

12. Brooks L, Shaw A, Sharp D, et al. Towards a better understanding of patients' perspectives of antibiotic resistance and MRSA: a qualitative study. Fam Pract 2008;25:341-8.

13. Brookes-Howell L, Elwyn G, Hood K, et al. 'The body gets used to them': patients' interpretations of antibiotic resistance and the implications for containment strategies. J Gen Intern Med 2012;27:766-72

14. Barden LS, Dowell SF, Schwartz B, et al. Current attitudes regarding use of antimicrobial agents: results from physician's and parents' focus group discussions. Clin Pediatr 1998;37:665-71.

15. Ingram J, Cabral C, Hay AD, et al. Parents' information needs, selfefficacy and influences on consulting for childhood respiratory tract infections: a qualitative study. BMC Fam Pract 2013;14:1-9.

16. Holstein BE, Andersen A, Krølner R, et al. Young adolescents use of medicine for headache: sources of supply, availability and accessibility at home. Pharmacoepidemiol Drug Saf 2008;17:406-10.

17. Holmström IK, Bastholm-Rahmner P, Bernsten C, et al. Swedish teenagers and over-the-counter analgesics - responsible, casual or careless use. Res Social Adm Pharm 2014;10:408-18.

18. Vodicka TA, Thompson M, Lucas $\mathrm{P}$, et al. Reducing antibiotic prescribing for children with respiratory tract infections in primary care: a systematic review. Br J Gen Pract 2013;63:445-54.

19. e-Bug: a place to play games and learn about microbes. 2014 www. e-bug.eu

20. Ajzen I. The theory of planned behavior. Organ Behav Hum Decis Process 1991;50:179-211.

21. Braun V, Clarke V. Using thematic analysis in psychology. Qual Res Psychol 2006;3:77-101.

22. Ziebland S, McPherson A. Making sense of qualitative data analysis: an introduction with illustrations from DIPEx (personal experiences of health and illness). Med Educ 2006;40:405-14.

23. Department for Communities and Local Government. English indices of deprivation 2015. 2015 https://www.gov.uk/government/statistics/ english-indices-of-deprivation-2015

24. Harvey K, Churchill D, Crawford $P$, et al. Health communication and adolescents: what do their emails tell Us? Fam Pract 2008;25:304-11.

25. Churchill R, Allen J, Denman S, et al. Do the attitudes and beliefs of young teenagers towards general practice influence actual consultation behaviour? Br J Gen Pract 2000;50:953-7.

26. Carlisle J, Shickle D, Cork M, et al. Concerns over confidentiality may deter adolescents from consulting their doctors. A qualitative exploration. J Med Ethics 2006;32:133-7.

27. Kitzinger J. The methodology of focus groups: the importance of interaction between research participants. Sociology of Health and IIIness 1994;16:103-21.

28. Norris $\mathrm{P}$, Chamberlain K, Dew K, et al. Public beliefs about antibiotics, infection and resistance: a qualitative study. Antibiotics 2013;2:465-76.

29. Reynolds L, McKee M. Factors influencing antibiotic prescribing in China: an exploratory analysis. Health Policy 2009;90:32-6.

30. McNulty CA, Boyle P, Nichols T, et al. Don't wear me out--the public's knowledge of and attitudes to antibiotic use. J Antimicrob Chemother 2007;59:727-38. 
31. Azevedo MM, Pinheiro C, Yaphe J, et al. Portuguese students' knowledge of antibiotics: a cross-sectional study of secondary school and university students in Braga. BMC Public Health 2009;9:359.

32. Huang Y, Gu J, Zhang M, et al. Knowledge, attitude and practice of antibiotics: a questionnaire study among 2500 chinese students. BMC Med Educ 2013;13:163.

33. Cebotarenco N, Bush PJ. Reducing antibiotics for colds and flu: a student-taught program. Health Educ Res 2008;23:146-57.

34. Pinder R, Sallis A, Berry D, et al. Behaviour change and antibiotic prescribing in healthcare settings: literature review and behavioural analysis. . 2015 https://www.gov.uk/government/uploads/system/ uploads/attachment_data/file/405031/Behaviour_Change_for_ Antibiotic_Prescribing_-_FINAL.pdf

35. Hansen DL, Holstein BE, Hansen EH. "I'd rather not take it, but": young women's perceptions of medicines. Qual Health Res 2009;19:829-39.

36. Maxwell KA. Friends: the role of peer influence across adolescent risk behaviors.. J Youth Adolesc 2002;31:267-77.

37. Welschen I, Kuyvenhoven M, Hoes A, et al. Antibiotics for acute respiratory tract symptoms: patients' expectations, GPs management and patient satisfaction. Fam Pract 2004;21:234-7. 